\title{
Brain MR Segmentation through Fuzzy Expectation Maximization and Histogram Based K-Means
}

\author{
Soodabeh Safa, Behrouz Bokharaeian, and Ali Soleymani
}

\begin{abstract}
Expectation maximization algorithm has been extensively used in a variety of medical image processing applications, especially for detecting human brain disease. In this paper, an efficient and improved semi-automated Fuzzy EM based techniques for 3-D MR segmentation of human brain images is presented. FEM along with histogram based K-means in initialization step is used for the labeling of individual pixels/voxels of a 3D anatomical MR image (MRI) into the main tissue classes in the brain, Gray matter (GM), White matter (WM), CSF (Celebro-spinal fluid). FEM's membership function were estimated through a histogram-based method. The results show our proposed FEM-KMeans has better performance and convergence speed compare to histogram based EM.
\end{abstract}

Index Terms-Brain MRI segmentation, fuzzy expected maximization, histogram based k-mean.

\section{INTRODUCTION}

Automatic three-dimensional (3-D) segmentation of the magnetic resonance (MR) scans is a challenging problem that has received an enormous amount of attention lately. Quantitative analysis of signal intensity on MRI changes and their correlation with clinical finding provide important information to diagnose of many neurodegenerative and psychiatric diseases. After the enhancement of images and brain extraction, brain segmentation is the next level. As a result, brain segmentation is of great interest to many researches.

In the context of neuro-imaging, 3-D segmentation of white matter (WM), gray matter (GM), and cerebro-spinal fluid (CSF) are extremely important for quantitative analysis such as volume measurements. An accurate and robust tissue classification is the basis for many applications such as: quantitative measurement of tissue volume in normal and diseased brain, morphological analysis, or visualization. Manual or even semi-automatic, Classification performed by a trained expert is labor-intensive and hence impractical for processing large amounts of data, highly subjective, and non-reproducible [1]. The main difficulties found in the automatic segmentation of MR brain images are due to the fact that image intensities are not necessarily constant for each tissue class. Several methods have been developed for this purpose, Lee and his colleagues not only applied active contours for automatic segmentation [2] but also used

Manuscript received March 10, 2013; revised April 30, 2013.

Soodabeh Safa is with the University Putra Malaysia, Selangor, 43400 Malaysia (e-mail: soodabeh_safa@yahoo.com).

Behrouz Bokharaeian is with Complutense University de Madrid, 28040 Spain (e-mail: bokharaeian@gmail.com).

Ali Soleymani is with the Faculty of Information and Science Technology, Universiti Kebangsaan Malaysia, Selangor, 43600 Malaysia (e-mail: ali.soleymani@gmail.com). another method watershed transform [3]. Besides, Duta developed a fully automated active shape model for segmentation and interpretation of MR images [4]. Watershed transform is another method that have used by some researchers such as Lee and his colleagues [3].In addition to aforementioned methods, atlas-based segmentation and Learning vector quantization (LVQ) models are two other methods applied in brain MR segmentation[5], [6].

As the most natural framework for the design of such methods is statistical/ probabilistic, Bayesian approach is more suitable for such applications with anatomical/geometric constraints about the location of each class which may be specified as prior probabilities, The resulting estimation procedure is EM algorithm which is iterative; in general, it contains two steps that are repeated until convergence as follows: Estimating the segmentation (class label), given the intensity models (GMM) for each class and Estimating the intensity models (probability density function), given segmentation with an appropriate initialization step.

The EM algorithm has been used by a number of medical image processing researchers for different kinds of studies [7], [8].

Yongyue improved EM by using hidden Markov random field (HMRF) model in which state sequence estimated through observations [9]. Also, Fua and his colleagues proposed an automatic hybrid image segmentation model that integrates the statistical EM model and the spatial pulse coupled neural network (PCNN) for brain magnetic resonance imaging (MRI) segmentation [10].

Mounira suggested another approach on building statistical model from data itself, for normal brain MRI and including clustering tissue type [11]. Gu proposed another improvement on EM algorithm to estimate parameters of the prior probabilities and likelihood probabilities and the Segmentation is carried out by means of a MAP estimator [12]. Also, Quost proposed a fuzzy EM algorithm in clustering imprecise data using finite mixtures of Gaussians [13].

In this paper we applied Quost's proposed fuzzy EM model to 3d brain MR images along with improvement on initialization phase done by a histogram based on k-means algorithm and a histogram based membership function

\section{MethOD}

Many kinds of computerized analyses can be used to extract information from three dimensional MRI data of the human head such as atlas based and mathematical morphological method. Another categorization is manual, 
semi-automatic and automatic categories. Manual segmentation is possible but is time-consuming task and subject to operator variability .In this section, first we explain our preprocessing phase, and will mention our Fuzzy EM method and our histogram-based EM initialization procedure.

During the preprocessing phase, the brain images are aligned with each other and in the normalizing each subject's anatomy becomes roughly the same shape, we corrected them for differences in slice time acquisition, and smooth them spatially. Data is combined across individuals and use standard coordinate system. In order to compare different data from different scans and research subjects, all the brain images have to be in the same 3D space. In the normalization step we stretch and squeeze the shape of the images so that their anatomy roughly matches a standard template and also makes localizing our activations easier and more meaningful, by making individual voxels' locations in a given image file match up in a standard way to a particular anatomical location.

An EM algorithm is used in statistics for finding maximum Likelihood estimate of parameters in probabilistic models, where the model depends on unobserved hidden variables [14]. EM is an iterative method which alternates between performing an expectation (E) step, which computes an expectation of the log likelihood with respect to the current estimate of the distribution for the hidden variables, and a maximization (M) step, which computes the parameters which maximize the expected log likelihood found on the E-step. These parameters are then used to determine the distribution of the hidden variables in the next E step .The Classic EM algorithm is an iterative procedure to compute the Maximum Likelihood (ML ) estimate in the presence of missing or hidden data. In ML Estimation, the model parameter(s) are estimated for which the observed data are the most likely. In the expectation, or E-step, the missing data are estimated given the observed data and current estimate of the model parameters. The result is achieved using the conditional expectation, explaining the choice of terminology. In the M-step, the likelihood function is maximized under the assumption that the missing data are known. Convergence is assured since the algorithm is guaranteed to increase the likelihood at each iteration [15].

A fuzzy EM algorithm can be considered as follows [13]: The value $\mu_{\tilde{\mathrm{x}}}(\mathrm{x})$ can be interpreted as the degree of possibility in which the real value is taken by the random variable $x_{i}$ is $x$. We suppose that the membership function of a multidimensional fuzzy number produced by the membership functions of its components. The complete data sample is $(\tilde{y}, z)$.

Also, the probability of a fuzzy event definition may be applied to measure the observed data log-likelihood [16]:

$$
L(\psi)=\sum_{z} p(z ; \psi) \int g(y \mid z ; \phi) d y
$$

Therefore, the E-step consists in calculating

$$
Q\left(\psi, \psi^{\mathrm{q}}\right)=E_{\psi^{q}}\left[\log l_{c}(\psi) \mid \widetilde{y}, z\right]
$$

The conditional density of a continuous random variable $X$ with respect to a fuzzy event $\tilde{x}$ with fuzzy membership function $\mu_{\tilde{x}_{i}}$ is:

$$
g x(x \mid \widetilde{x})=\frac{\mu_{\tilde{x}}(x) g x}{\int \mu_{\tilde{x}}(x) g x d x}
$$

With the following notations:

$$
\begin{gathered}
p_{i}^{(q)}=p_{\text {(gq) }}\left(\tilde{x}_{i}\right)=\sum_{k=1}^{r} \pi_{k}^{(q)} \int \mu_{\tilde{x}_{i}}(x) g_{k}\left(x ; \psi^{(q)}\right) \cdot d x \\
\pi_{i j k}(q)=E_{(\xi q)}\left[x \mid \tilde{x}, z_{i k}=1\right]=\frac{\int x_{j} \mu_{\tilde{x}_{i j}}\left(x_{j}\right) g_{j k}\left(x_{j} ; \psi_{k}{ }^{(q)}\right) \cdot d x}{\gamma_{i j k}(q)}(5) \\
\varepsilon_{i j k}(q)=E_{\psi^{(q)}}\left[x_{i j}^{2} \mid \widetilde{x}_{i}, z_{i k}=1\right]=\frac{\int x_{j}^{2} \mu_{\tilde{x}_{i j}}\left(x_{j}\right) g_{j k}\left(x ; \psi^{(q)}\right) \cdot d x}{\gamma_{i j k}(q)}(6) \\
\gamma \stackrel{(q)}{(q)}=p_{\psi^{(q)}}\left(x^{\sim}{ }_{i} \mid z_{i k}=1\right)=\int \mu_{\tilde{x}_{i}}(x) g_{k}\left(x ; \psi^{(q)}\right) \cdot d x(7)
\end{gathered}
$$

And using Bayes' theorem, we have:

$$
t \stackrel{(q)}{i k}=E_{\psi(q)}\left(z_{i k} \mid x_{i}^{\sim}\right)=\frac{p_{\psi}(q)\left(x^{\sim}{ }_{i} \mid z_{i k}=1\right)}{p_{\psi}(q){ }\left(x^{\sim}{ }_{i}\right)}=\frac{\gamma_{i, k}^{(q)} \pi_{k}^{(q)}}{p_{i}^{(q)}}
$$

The E-step now consists in computing [9]:

$$
\begin{aligned}
Q\left(\psi, \psi^{\mathrm{q}}\right)=\sum_{k=1}^{g} \log \pi_{k} \sum_{i=1}^{n} t_{i k}^{(q)}-\frac{n p}{2 \log (2 \pi)} & \\
& -\sum_{k=1}^{g} \sum_{j=1}^{p} \log \sigma_{j k}^{(q)} \sum_{i=1}^{n} t_{i k}^{(q)} \\
& -1 / 2 \sum_{i=1}^{n} \sum_{k=1}^{g} t_{i k}^{(q)}\left(\sum_{j=1}^{p} \frac{1}{\sigma_{j k}^{(q)^{2}}} \varepsilon_{i, j, k}^{(q)}\right. \\
& -2 \sum_{j=1}^{p} \frac{m_{j k}^{(q)}}{\sigma_{j k}^{(q)} \pi_{i j k}^{(q)}}+\sum_{j=1}^{p} \frac{m_{j k}^{(q)^{2}}}{\sigma_{j k}^{(q)}}
\end{aligned}
$$

M-step:

$$
\begin{gathered}
\pi_{k}^{(q+1)}=\frac{1}{n} \sum_{k=0}^{n} \mathrm{t}_{\mathrm{ik}}^{(\mathrm{q})} \\
m_{j k}^{(q+1)}=\frac{\sum_{i=1}^{n} \mathrm{t}_{\mathrm{ik}}^{(\mathrm{q})} \mu_{i j k(q)}}{\sum_{i=1}^{n} \mathrm{t}_{\mathrm{ik}}^{(\mathrm{q})}} \\
\sigma_{k}^{(q+1)}=\sqrt{\frac{\sum_{i=1}^{n} t_{i k}^{(q)} \sum_{j=1}^{p}\left(\varepsilon_{i j k}^{(q)}-2 m_{j k}^{(q)} \pi_{i j k}^{(q)}+m_{j k}^{(q) 2}\right)+\lambda_{k}}{p \sum_{i=1}^{n} t_{i k}^{(q)}+p\left(m_{0}+p+1\right)}}
\end{gathered}
$$

$Q$ Stands for iteration. Initial value for $q=1$.

Comparing class EM with crisp data shows that the crisp quantities $x_{i, j}$ and $x_{i, j}^{2}$ are replaced with the conditional expectations $\pi_{i j k}$ and $\pi_{i j k}$ of the fuzzy variables $\tilde{x}_{i j}$ and $\tilde{x}_{i j}{ }^{2}$, respectively [13].

To calculate the membership function in the previous equations we used a simple model of Dou's method for the MR images [17]. For simplicity we applied Dou's proposed steps: calculating the histogram of whole MRI sequence and transforming the histogram into membership function by selecting some feature values such as peaks and valleys of the histogram.

If $G L_{K}$ presents $k_{t h}$ gray level, and the total number of gray level is $\mathrm{L}, k=0,1 . \ldots L-1$, the histogram $P^{\left(G L_{K}\right)}$ gives us estimation of the probability of $G L_{K}$

$$
P\left(G L_{K}\right)=\frac{n_{K}}{N}
$$

$n_{k}$ refers to the total number of pixel which their gray level is equal to $G L_{K}$.

$\left(G L_{S I}^{p k}, \mathrm{p}\left(G L_{S I}^{p k}\right)\right)$ are noted as peaks as the features points for transformation and their valleys, considered 
as $\left(G L_{S I}^{v l}, \mathrm{p}\left(G L_{S I}^{\nu l}\right)\right)$. Let $F$ denote one of transformation Operation, the membership function is presented as equation 14.

$$
\mu_{\text {TisS }}^{S I}=F\left\{\left(G L_{S I}, P\left(G L_{S I}\right)\right), p\left(G L_{S I}^{p k}\right), p\left(G L_{S I}^{v l}\right)\right\}
$$

where

$$
G L_{S I}=\left\{G L_{T 1}, G L_{T 2}\right\}
$$

For example, $\mu_{\text {Tiss }}^{S I}$ can be obtained with the properties ranking in $\mathrm{T} 1$,

$$
\begin{aligned}
& \mu_{W M}^{T 1} \propto G L_{T 1} ; \text { and } \mu_{C S F}^{T 1} \propto \frac{1}{G L_{T 1}} \\
& \mu_{G M}^{T 1} \propto\left\{\begin{array}{l}
G L_{T 1}, I F G L_{T 1} \leq G L_{T 1}^{P^{K}} \\
\frac{1}{G L_{T 1}}, I F G L_{T 1}^{P^{K}}<G L_{T 1}
\end{array}\right.
\end{aligned}
$$

Example of histogram and membership function for one sample MRI image is given in the Fig. 1.

Another improvement in our proposed algorithm was in initialization phase. EM algorithm can be initiated with different methods. Initialization means finding mixture model parameters $\left(\pi_{i}, m_{i}, \sigma_{i}\right)\left(\left(\mu_{i}, \sigma_{\mathrm{i}}, c_{i}\right)\right.$ in classic notation). These parameters can be obtained using various techniques such as: a) guess or experience, b) Histogram (with plotting histogram), c) K-means clustering algorithm.

We ran algorithm with two different initialization methods: classic histogram and a histogram-based k-means. Based on results, histogram based k-means shows better performance.

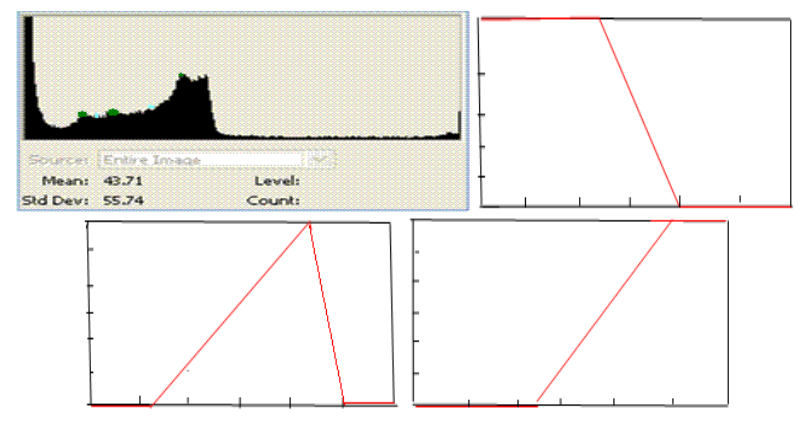

$$
\text { Histogram } \mu_{C S F}^{T 1} \mu_{G M}^{T 1} \mu_{W M}^{T 1}
$$

Fig. 1. Histogram of a sample MRI image (left) and continuous membership function (Right)

\section{RESULTS}

This section provides simulation results on MRI human brain data set. It also provides some performance analysis of EM algorithm with and without K-means initialization. Computational steps are explained through examples. Synthetic One dimensional data set and Gaussian Mixture Model using EM algorithm Data Analysis results using histogram, K-Means and EM algorithms on Real MR image data set from Harvard University (Massachusetts General Hospital, Center for Morphometric Analysis Internet Brain Segmentation Repository) (IBSR) which has MRI data base for doing scientific study on human brain on different subjects and has been considered during this project. Data set consists twenty Normal Subjects, T1-Weighted Scans with
Segmentations: 16-bit MR data files or 16-bit MR data files or first half 16-bit MR data files or 8-bit MR data files or 8-bit brain-only MR data files and CSF/gray/white/other manual segmentation files OR 8-bit that can analyze format raw images and segmentations.An example of the implementation of the method is shown in the following figures. Fig. 2 presents three representative slice of a normal T1 MR image of a brain; Fig. 3 display the White matter after segmentation and Fig. 4 shows grey matter after FEM-KMeans segmentation process. Besides the classic EM algorithm segmented MR image has been shown at Fig. 5.In the mentioned classic EM algorithm $\pi_{k}^{0}$ initialized to random, $m_{j k}^{0}$ to identity matrix and $\sigma_{k}^{0}$ to $\frac{1}{c}$. The comparison show a significant correspondence. Segmented images by our proposed FEM-KMeans are more accurate in comparison with classic EM algorithm. Evaluation of the statistical differences of results was performed by a Wilcoxon signed rank test for paired data with manual ground truth. The result indicates that our FEM k-means method has better performance and statistically less difference with manual ground compare to classic EM.

Furthermore our results shows using histogram in finding initial centroids in K-means algorithm in initialization phase of developed FEM algorithm makes faster convergence in compare to other previously used methods. The entire implementation of segmentation has been done using Matlab version 7.0 beside Image processing toolbox and MRIcro software version 1.2.The MR image format was DICOM and the resolutions were $256 \times 256$.

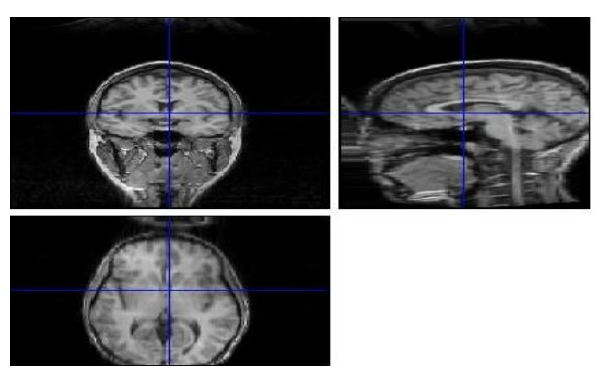

Fig. 2. Normal T1 MR image
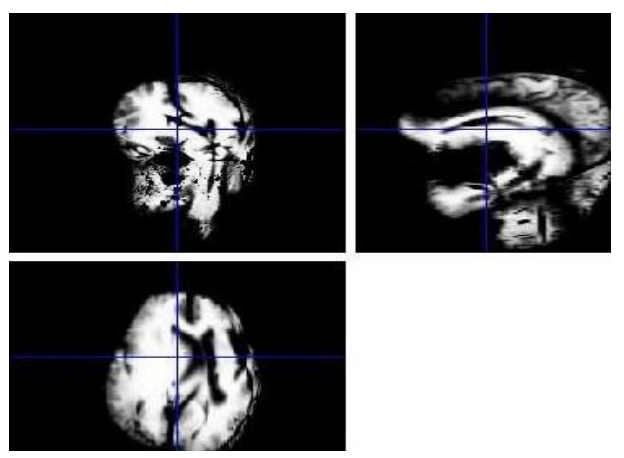

Fig. 3. White matter

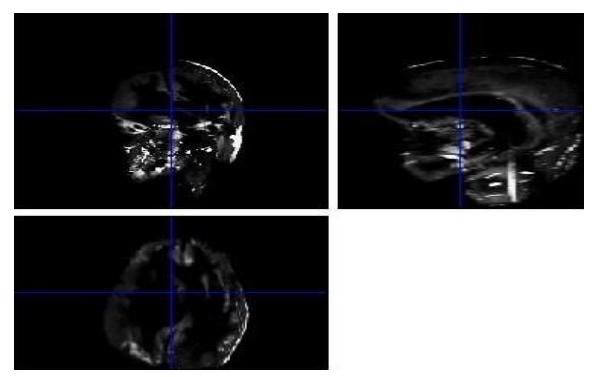

Fig. 4. Gray matter 

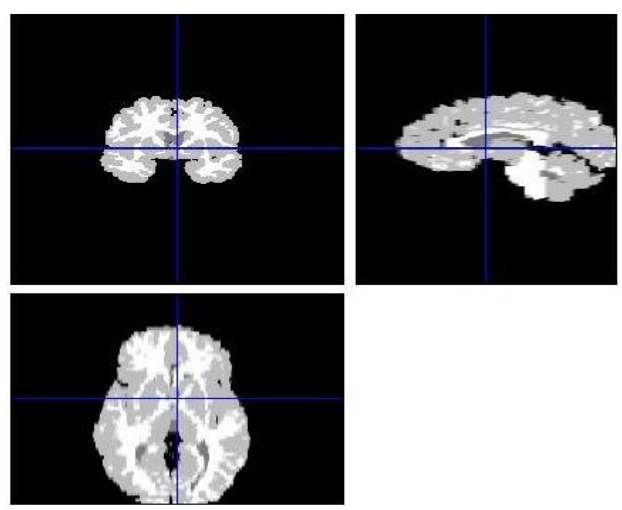

Fig. 5. MR Segmentation by classic EM method

\section{CONCLUSION AND Future Works}

Quantitative analysis of signal intensity on MRI changes and their correlation with clinical finding provides important information to diagnose, and brain segmentation is of great interest to many researches. In this paper, a fuzzy expectation maximization method along with histogram based K-means is used for the labeling of individual pixels/voxels of a 3D anatomical MR image with the help of mixture models. A histogram-based method is employed for estimating the membership function. The results indicate that our proposed FEM-KMeans has better performance and convergence speed compare to classic EM and EM-histogram.

In this study, Fuzzy logic is used to cluster MR images, other Fuzzy based methods will be applied in the segmentation of MR images. Furthermore, augmenting Fuzzy-k-means in initialization step along with Fuzzy-EM and employing better Fuzzy membership function scan improve results. These are the issues that future research may assist.

\section{REFERENCES}

[1] M. A. Balafar, A. R. Ramli, M. I. Saripan, and S. Rhaomholir, "Review of brain MRI image segmentation methods," Artificial Intelligence Review, vol. 33, pp. 261-274, 2010.

[2] H. Li, A. Yezzi, and L. D. Cohen, "3D brain segmentation using dual-front active contours with optional user interaction," International Journal of Biomedical Imaging, pp. 1-17, 2006.

[3] N. Li, M. Liu, and Y. Li , "Image segmentation algorithm using watershed transform and level set method," in Proc. IEEE international conf. on acoustics, speech and signal processing, pp. 613-616, 2007.

[4] N. Duta and M. Sonka, "Segmentation and interpretation of MR brain images: An improved active shape model," IEEE Trans. on Medical Imaging, vol. 17, no. 6, pp. 1049-1062, December 1998.

[5] T. Rohlfing et al., "Evaluation of atlas selection strategies for atlas-based image segmentation with application to confocal microscopy images of bee brains," Journal of NeuroImage, vol. 21, no. 4, pp. 1428-1442, 2004.

[6] D. Tian and L. Fan, "A brain MR images segmentation method based on SOM neural network," in Proc. the 1stinternational conf. on bioinformatics and biomedical engineering, pp. 686-689, 2007.

[7] H. Khotanlou, "3D brain tumors and internal brain structures segmentation in MR image," Ph.D. Dissertation, Paris Institute of Technology, 2008.

[8] M. Lynch, D. Ilea, K. Robinson, O. Ghita, and P. F. Whelan, "Automatic seed initialization for the expectation-maximization algorithm and its application in 3D medical imaging," vol. 31, no. 5 , pp. 332-340, 2007.

[9] Z. Yongyue, M. Brady, and S. Smith, "Segmentation of brain MR images through a hidden markov random field model and the expectation-maximization algorithm," IEEE Trans. on Medical Imaging, vol. 20, no. 1, January 2001.
[10] J. C. Fua, C. C. Chenb, and J. W. Chaib, "Image segmentation by EM-based adaptive pulse coupled neural networks in brain magnetic resonance imaging," Computerized Medical Imaging And Graphic , vol. 34, pp. 308-320, 2010.

[11] R. Mounira and M. Salah Medjram, "Brain MRI segmentation and lesions detection byemalgorithm," World Academy of Science, Engineering and Technology, pp. 139-142, 2006.

[12] D. B. Guand and J. X. Sun, "EM image segmentation algorithm based on an inhomogeneous hidden MRF model," in IEEE Proc. Image Signal Process, vol. 152, no. 2, April 2005.

[13] B. Quost and T. Denoeux, "Clustering fuzzy data using the fuzzy EM algorithm," in SUM'10 Proc. of the 4th International Conf. on Scalable Uncertainty Management, pp. 333-346, 2010.

[14] C. Carson, S. Belongie, and H. Greenspan, "Blob world: Image segmentation using expectation-maximization and its application to image querying," IEEE Trans. Pattern Analysis and Machine Intelligence, vol. 24, no. 8, pp. 1026-1038, August 2002.

[15] N. Radford, H. Geoffrey, and M. I. Jordan, "A view of the EM algorithm that justifies incremental, sparse, and other variants," in Learning in Graphical Models, Cambridge, MA: MIT Press, 1999, pp. $355-368$

[16] H. J. Zimmermann, Fuzzy set theory and its applications, Springer publishing, 2001, pp. 130-132.

[17] W. Dou, Y. Ren, Y. Chen, S. Ruan, and D. Bloyet, "Histogram-Based generation method of membership function for extracting features of brain tissues on MRI images," Lecture Notes in Computer Science, 2005, vol. 3613, pp. 189-194, 2005.

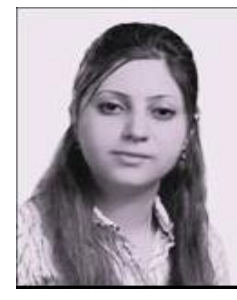

Soodabeh Safa received her B.Sc. in software engineering, Mazandaran University of science and technology(2000-2004), M.Sc. in computer science, Multimedia University, Malaysia(2008-2010). PhD student in content based image retrieval in University Putra Malaysia (2010) Faculty of Computer Science and Information Technology. E-mail: Soodabeh_safa@yahoo.com. She worked in an artificial intelligence group in Multimedia University in MRI brain segmentation. Now she is working in Multimedia Retrieval research group in University of Putra Malaysia. Ms Soodabeh Safa is a student member of IEEE and Member of the International Association of Computer Science and Information Technology (IACSIT) and a former Member of Computer Science and Engineering group Mazandaran University of science and technology in Iran. She worked as a corresponding author in Using Rotation Forest for Protein Fold Prediction Problem: An Empirical Study". (2010). Proceeding in: 8th European Conference on Evolutionary Computation, Machine Learning and Data Mining in Bioinformatics.

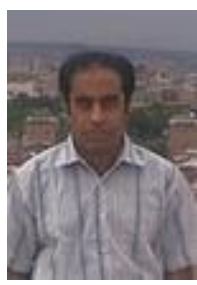

Behrouz Bokharaeian received his B.Sc. in software engineering, Sharif University Technology (1997-2001), M.Sc in Software engineering, Amirkabir University of Technology, (2001-2003). And also Master in Biomedical Informatics from Amirkabir university of Technology, (2005-2008), And Ph.D. student in Complutense University de Madrid (2011) Faculty of Computer Science and Information Technology E-mail: bokharaeian@gmail.com. He worked in an artificial group in Amirkabir University of Technology.

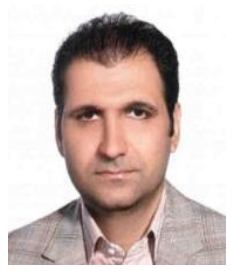

Ali Soleymani received his B.Sc. in Computer Hardware Engineering and M.Sc. in Computer Architecture Engineering both from Islamic Azad University, Iran in 2002 and 2006 respectively. Then he was a lecturer in Islamic Azad University, Iran from 2003 to 2010 and now he is a Ph.D student in the Faculty of Information Science and Technology, University Kebangsaan Malaysia (UKM) since 2010. His research interests include image processing, cryptography and network security. Email: ali.soleymani@gmail.com. 